\title{
Mitosis-Coupled, Microtubule-Dependent Clustering of Endosomal Vesicles around Centrosomes
}

\author{
Hiroyuki Takatsu ${ }^{12^{*}}$, Yohei Katoh ${ }^{*}$, Tomoko Ueda ${ }^{1 *}$, Satoshi Waguri ${ }^{3}$, Takashi Murayama ${ }^{4}$, \\ Senye Takahashi' ${ }^{1}$,Hye-Won Shin ${ }^{1,2}$, and Kazuhisa Nakayama ${ }^{1 \dagger}$ \\ ${ }^{1}$ Graduate School of Pharmaceutical Sciences, Kyoto University, Sakyo-ku, Kyoto 606-8501, Japan, \\ ${ }^{2}$ Career-Path Promotion Unit for Young Life Scientists, Kyoto University, Sakyo-ku, Kyoto 606-8501, Japan, \\ ${ }^{3}$ Department of Anatomy and Histology, Fukushima Medical University School of Medicine, Fukushima City, \\ Fukushima 960-1295, Japan, ${ }^{4}$ Department of Pharmacology, Juntendo University School of Medicine, \\ Bunkyo-ku, Tokyo 113-8421, Japan
}

\begin{abstract}
$A B S T R A C T$. Upon cell division, not only cells themselves but also their organelles undergo drastic shape changes, although the behaviors of organelles other than the Golgi apparatus remain poorly understood. We followed the spatiotemporal changes in the localization of transferrin receptor ( $\mathrm{TfnR}$ ) and other proteins. In early mitotic phases, a population of proteins cycling through the endocytic recycling compartment (ERC) exhibits a distinct spatiotemporal change from that of Golgi proteins. In prophase/prometaphase, when the cell surface-to-volume ratio is reaching its minimum, the ERC proteins are transiently assembled around the centrated centrosome in a microtubule- and dynein-dependent manner, and soon separated polewards into two clusters concomitant with separation of duplicated centrosomes. Electron microscopic analysis revealed that endosomal vesicles containing endocytosed transferrin cluster tightly around centrosomes without fusing with one another. As cytokinesis proceeds, the clusters gradually collapse, and the ERC proteins reassemble around the furrowing equatorial region. FRAP (fluorescence recovery after photobleaching) analyses of EGFP-TfnR-expressing cells revealed minimal membrane exchange between the endosomal clusters and other cellular compartments until anaphase/ telophase, when membrane traffic resumes. Our observations indicate that ERC clustering around centrosomes plays a fundamental role in restricting membrane delivery to the plasma membrane during early mitotic phases, when the cell surface-to-volume ratio reaches its minimum.
\end{abstract}

Key words: recycling endosome, transferrin receptor, spindle pole, cytokinesis, dynein

Cell division entails local delivery and retrieval of lipids and proteins via membrane trafficking, to coordinate the drastic shape and size changes associated with mitosis. Furthermore, upon symmetric cell division, each organelle must be equally allocated into the two daughter cells; therefore, organelles also undergo dynamic morphological changes (Fürthauer and González-Gaitán, 2009; Montagnac

\footnotetext{
*H. Takatsu, Y. Katoh, and T. Ueda contributed equally to this work. †To whom correspondence should be addressed: Kazuhisa Nakayama, Graduate School of Pharmaceutical Sciences, Kyoto University, Sakyo-ku, Kyoto 606-8501, Japan.

E-mail: kazunaka@pharm.kyoto-u.ac.jp Abbreviations: CReSCent, cluster of recycling compartment around spindle pole centrosomes; DMT1, divalent metal transporter 1; EGFP, enhanced green fluorescent protein; ERC, endocytic recycling compartments; FRAP, fluorescence recovery after photobleaching; HRP, horseradish peroxidase; LactC2, lactadherin C2 domain; PS, phosphatidylserine; Tfn, transferrin; TfnR, transferrin receptor; TGN, trans-Golgi network.
}

et al., 2008; Wei and Seemann, 2009). For example, in interphase cells, the Golgi apparatus is located in the perinuclear region, but in prophase/prometaphase it is fragmented and/or absorbed into the endoplasmic reticulum (AltanBonnet et al., 2004; Colanzi et al., 2003; Shorter and Warren, 2002). In each daughter cell part, two Golgi assemblies are formed in telophase, one near the spindle pole and the other near the minus end of the central spindle; these join each other to reconstitute a single Golgi stack as cytokinesis proceeds to completion (Gaietta et al., 2006; Seemann et al., 2002). In contrast, the behaviors of other organelles during cell division have been poorly characterized. Endocytic compartments are thought to undergo ordered partitioning similar to that of the Golgi (Dunster et al., 2002; Montagnac et al., 2008, 2009; Schweitzer et al., 2005; Simon et al., 2008), although this process has not been precisely described.

In this study, we examined the localization of various 
marker proteins during mitosis. We found that in early mitotic phases, proteins transported through the endocytic recycling compartment (ERC) exhibit a characteristic spatiotemporal change, quite different from that of Golgiresident proteins. In prophase, a tight cluster of endosomal vesicles is transiently formed in a microtubule- and dyneindependent manner around the centrosome at the cell center. This transient structure is subsequently separated into two clusters as the duplicated centrosomes are separated polewards. The endosomal clusters undergo minimal membrane exchange with other membrane compartments until anaphase/ telophase, indicating that membrane traffic to and from these endosomal clusters is suppressed until that time. These observations support the view that the endosomal clustering plays a fundamental role in temporal restriction of membrane delivery to the plasma membrane during early phases of mitosis, when the cell surface-to-volume ratio reaches its minimum.

\section{Materials and Methods}

\section{Antibodies and reagents}

Sources of antibodies used in the present study were as follows: monoclonal mouse anti-GalT (Kawano et al., 1994), provided by Tatsuo Suganuma (University of Miyazaki); polyclonal rabbit antiTGN46 (Kain et al., 1998), provided by Minoru Fukuda (SanfordBurnham Medical Research Institute); monoclonal rat anti- $\alpha$ tubulin, Abcam; monoclonal mouse anti- $\beta$-tubulin, Millipore; monoclonal mouse anti- $\gamma$-tubulin, Sigma-Aldrich; monoclonal mouse anti-TfnR, Zymed; polyclonal rabbit anti-giantin, COVANCE; polyclonal rabbit anti-NuMA, Cell Signaling; monoclonal mouse anti-EEA1, anti-Lamp-1, anti-syntaxin 6, and anti-Vti1a, BD Biosciences; AlexaFluor-conjugated Tfn and secondary antibodies, Molecular Probes; horseradish peroxidase-conjugated Tfn, Jackson ImmunoResearch Laboratories; nocodazole and S-trityl-L-cysteine, Sigma-Aldrich.

\section{Plasmids}

Expression vectors for human DMT1-I and -II tagged N-terminally with enhanced green fluorescent protein (EGFP) (Tabuchi et al., 2002) were provided by Mitsuaki Tabuchi (Kagawa University). An expression vector for human TfnR tagged C-terminally with EGFP was constructed by subcloning the entire coding sequence of human TfnR into the pEGFP-N3 vector (Clontech). An expression vector for FLAG-tagged dynamitin (Hirose et al., 2004) was provided by Mitsuo Tagaya (Tokyo University of Pharmacy).

A LactC2 cDNA fragment (Uchida et al., 2011) (provided by Hiroyuki Arai, University of Tokyo) was initially subcloned into pcDNA3-mRFP (provided by Roger Tsien). The mRFP-LactC2 cDNA fragment was then amplified by polymerase chain reaction from pcDNA3-mRFP-LactC2 and cloned into the pGEX-6P-1 vector (GE Healthcare).
Plasmids for production of replication-defective, self-inactivating lentiviral vectors, pRRLsinPPT, and packaging plasmids (pRSVREV, pMD2.g, and pMDLg/pRRE) (Thomas et al., 2009) were kindly provided by Peter McPherson (McGill University). A destination cassette from pcDNA3.2/V5-DEST (Invitrogen) was inserted into pRRLsinPPT in order to convert it to a Gateway system destination vector, and the resultant plasmid was named pRRLsinPPT-DEST. A plasmid vector for lentiviral production of human TfnR tagged with MEF (Myc-TEV-Flag; provided by Toshiaki Isobe, Tokyo Metropolitan University) (Ichimura et al., 2005) and EGFP was described previously (Takahashi et al., 2012). The resulting plasmid, pRRLsinPPT-MEF-TfnR-EGFP, was used for lentiviral vector production.

\section{Lentiviral vector production and establishment of cell lines stably expressing MEF-TfnR-EGFP}

pRRLsinPPT-MEF-TfnR-EGFP was transfected into HEK293FT cells (Invitrogen) using Polyethylenimine Max (Polysciences) together with the packaging plasmids (pRSV-REV, pMD2.g, and $\mathrm{pMDL} / \mathrm{pRRE}$ ). Transfection mix was replaced with fresh culture medium at $8 \mathrm{hr}$ after transfection. Samples of culture medium containing the lentiviral vector were collected at 24,36 , and $48 \mathrm{hr}$ after transfection, passed through a $0.45 \mu \mathrm{m}$ filter (Millipore), and centrifuged at $32,000 \times \mathrm{g}$ at $4{ }^{\circ} \mathrm{C}$ for $4 \mathrm{hr}$ using an $\mathrm{R} 15 \mathrm{~A}$ rotor and Himac CR22G centrifuge (Hitachi Koki). The precipitated viral vector was resuspended in minimal essential medium.

A HeLa cell line stably expressing MEF-TfnR-EGFP, HeLa (MEF-TfnR-EGFP), was established as described previously (Takahashi et al., 2012).

\section{Preparation of recombinant mRFP-LactC2}

The mRFP-LactC2 fused to the C-terminus of glutathione $S$ transferase were expressed in Escherichia coli BL21-Codon Plus (DE3) cells (Stratagene) by treatment with $0.1 \mathrm{mM}$ Isopropyl $\beta$-D-thiogalactopyranoside overnight at $20^{\circ} \mathrm{C}$. The protein was purified in a single step on a glutathione-Sepharose 4B column followed by cleavage of the GST portion with PreScission protease (GE Healthcare).

\section{Cell culture, RNA interference, immunofluorescence analysis, and time-lapse recording}

HeLa cells cultured in minimal essential medium supplemented with $10 \%$ fetal bovine serum were transfected with an appropriate expression vector(s) using the FuGENE6 transfection reagent (Roche Applied Science) and incubated for 24-48 hr before analyses. For immunofluorescence analyses, cells were fixed, permeabilized, and stained with antibodies as described previously (Shin et al., 2005, 2004), and observed with an Axiovert $200 \mathrm{M}$ microscope or a PASCAL confocal microscope (Carl Zeiss). For time-lapse analyses, an A1R-MP confocal microscope (Nikon) was used. Images acquired were analyzed using MetaMorph (Molecular Devices) software. High-speed confocal microscopy was performed 
using a Nipkow disc confocal laser scanning unit (Yokogawa, CSU22) as described previously (Kobayashi and Murayama, 2009). For FRAP (fluorescence recovery after photobleaching) analyses, cells were incubated in HEPES-buffered modified Eagle's medium and placed on a microscope stage that had been prewarmed at $37^{\circ} \mathrm{C}$. The cells were observed and bleached using an FV1000D confocal system on an inverted IX81 microscope (Olympus). To visualize FRAP of TfnR-EGFP, images were acquired sequentially every $30 \mathrm{sec}$.

\section{Electron microscopy}

Incubation of cells with HRP-conjugated transferrin, and subsequent incubation with diaminobenzidine and $\mathrm{H}_{2} \mathrm{O}_{2}$ were performed as described previously (Takahashi et al., 2012). Samples were fixed with $2 \%$ glutaraldehyde- $2 \%$ paraformaldehyde in $0.1 \mathrm{M}$ phosphate buffer ( $\mathrm{pH} 7.2$ ), and processed for conventional electron microscopy as described previously (Waguri et al., 1999).

\section{Results}

\section{Accumulation around centrosomes of proteins trafficking through the ERC}

By comparing localizations of a variety of organelle markers with those of Golgi proteins in various mitotic phases, we found that transferrin receptor ( $\mathrm{TfnR}$ ) exhibits a characteristic spatiotemporal change that is completely different from that of a Golgi protein, giantin (Fig. 1A; also see Fig. 7). In a large fraction of mitotic HeLa cells, a TfnR-positive cluster is transiently formed around the centrated centrosome in prophase, and is subsequently separated into two clusters (green arrows) as the duplicated centrosomes are separated into the two hemispheres. As cytokinesis proceeds, the clusters gradually collapse. In contrast, the Golgi apparatus, depicted by giantin staining, remains disassembled/fragmented prior to the onset of cytokinesis, when it forms two assemblies in each dividing cell part, one near the spindle pole and the other near the minus end of the central spindle (red arrows) (Gaietta et al., 2006; Seemann et al., 2002). A $\mathrm{TfnR}$ chimera, in which TfnR is fused to EGFP, also forms such clusters around spindle poles when expressed in HeLa cells (Fig. 1B and Video S1). Time-lapse analysis of cells stably expressing TfnR-EGFP showed that a TfnR-EGFPpositive cluster is very transiently formed at the cell center in prophase and is soon separated polewards into two clusters (Fig. 1C and Video S2); the initial clustering appears to serve as a cue for the subsequent separation (see Discussion). Next, the clusters near the spindle poles gradually collapse as new assemblies are formed around the furrowing equatorial region (Video S3, and Video S4, left). Furthermore, three-dimensional time-lapse imaging revealed that the initial formation of the TfnR-EGFP-positive cluster at the cell center, as well as the subsequent commencement of separation into two clusters, takes place as the cell approaches a spherical shape (Fig. 1D and Video S3). Clustering of TfnR around mitotic centrosomes is also detectable in various cell lines, including monkey kidney COS-1 cells, human cervical epithelial HEp-2 cells, human hepatoma Huh-7 cells, and rat Clone 9 hepatocytes (Fig. S1).

We examined the distribution of various Golgi and endosomal proteins during cell division, but failed to detect accumulation around mitotic centrosomes of many Golgi and endosomal proteins, including $\beta$-COP, GM130, GalT, golgin-245, EEA1, and Lamp-1 (Fig. 2). On the other hand, Vtila, syntaxin 6 , syntaxin 16 , and TGN46 were often clustered around centrosomes (Fig. 2). The former three proteins are Q-SNAREs involved in retrograde transport from the ERC to the trans-Golgi network (TGN) (Mallard et al., 2002; Nishimoto-Morita et al., 2009); TGN38/46 is endocytosed from the cell surface to the TGN through the ERC (Ghosh et al., 1998; Maxfield and McGraw, 2004), although at steady state it is predominantly found on the TGN. We therefore reasoned that the TfnR cluster around each spindle pole represents a subpopulation or derivative of the ERC.

To confirm that the cluster is indeed derived from the ERC, we exploited a recent observation that phosphatidylserine (PS) is enriched in membranes of the ERC (Uchida et al., 2011); PS was detected using a PS-specific probe, the $\mathrm{C} 2$ domain of lactadherin (LactC2). As shown in Fig. 3A, throughout the phases of mitosis, the TfnR-EGFP signals almost completely overlap with those labeled with mRFP-LactC2, although mRFP-LactC2 also labels other intracellular structures. To further confirm that the clusters around mitotic centrosomes represent the ERC, we compared the distributions of the type I and type II isoforms of divalent metal transporter 1 (DMT1) during mitosis. The DMT1-I and -II isoforms are generated via alternative splicing of the 3'-terminal exon, which contains an ironresponsive element; the two isoforms differ from each other only in the cytoplasmic tail sequence. DMT1-I and -II are localized primarily to late endosomes/lysosomes and the ERC, respectively (Tabuchi et al., 2002); DMT1-II is responsible for transport across endosomal membranes of iron internalized via the Tfn-TfnR complex (Tabuchi et al., 2002). As shown in Fig. 3, B and C, EGFP-DMT1-II formed clusters that overlapped completely with those of TfnR (B), whereas EGFP-DMT1-I remained scattered as cytoplasmic puncta in early mitotic phases (C). Taken together, these data suggest that the cluster around each spindle pole centrosome represents a subpopulation of the ERC. Hereafter, we refer to the clustered structure as CReSCent (for Cluster of Recycling compartments around Spindle pole Centrosome).

\section{CReSCent is inactive in terms of membrane traffic}

We next characterized the CReSCent from the viewpoint 


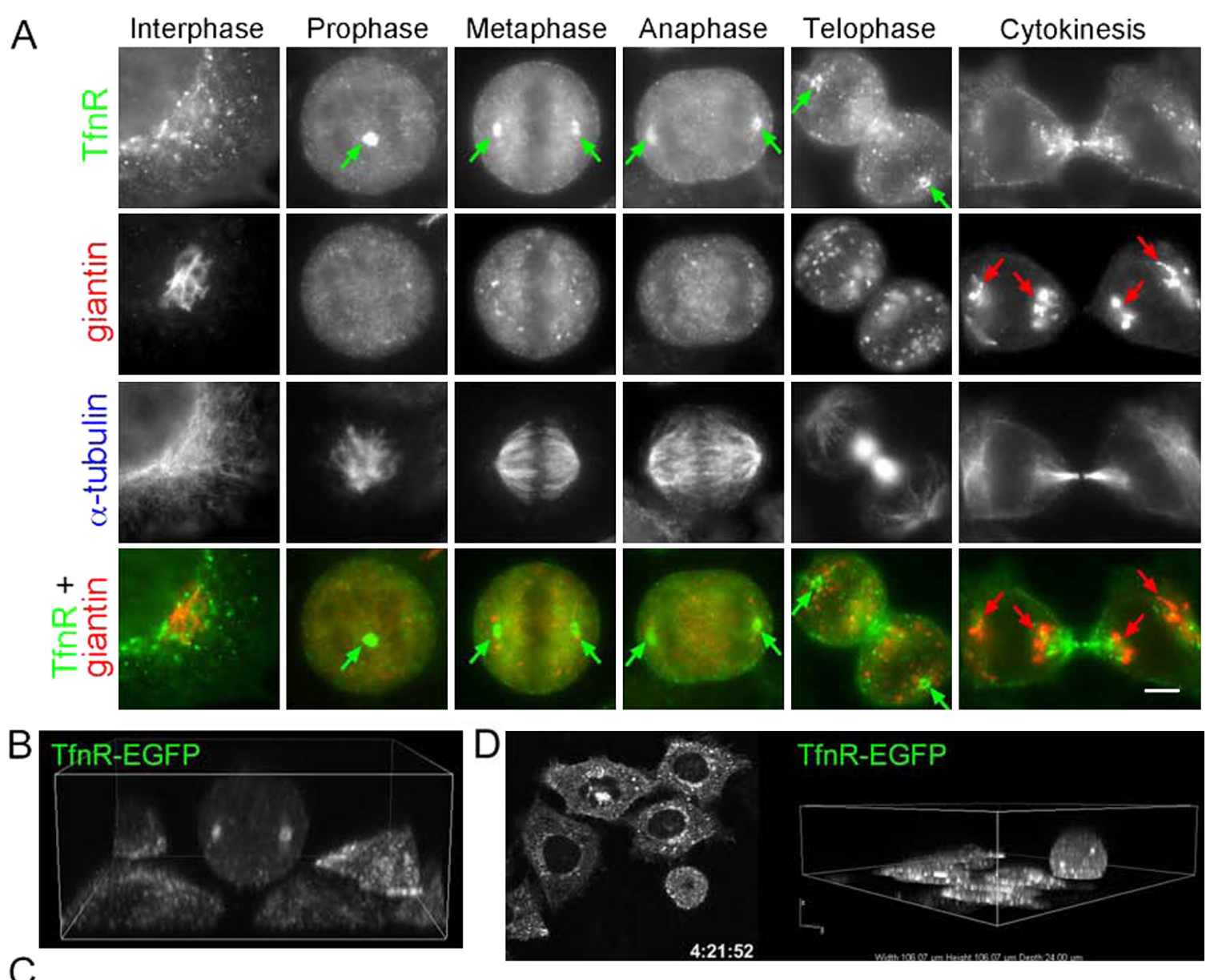

C

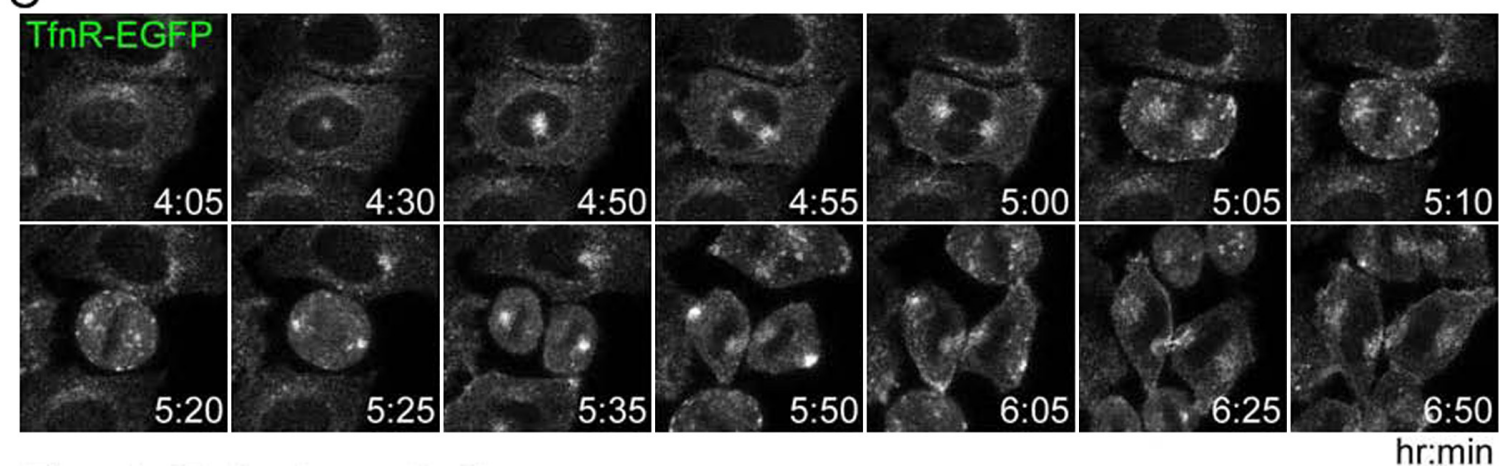

Fig. 1. Transient localization of TfnR to the CReSCent structures during mitosis. (A) Comparison of spatiotemporal changes in the distributions of TfnR and giantin. HeLa cells were triple-stained for TfnR, giantin and $\alpha$-tubulin. Images were acquired from cells in various mitotic phases and arranged in temporal order of the phases. Green arrows indicate positions of TfnR-positive structures around the centrosome/spindle poles from prophase through telophase. Red arrows indicate positions of giantin-positive structures around spindle poles and near the central spindle during cytokinesis. Bar, $5 \mu \mathrm{m}$. (B) Three-dimensional analysis of TfnR-EGFP localization. Using an A1R-MP confocal microscope, images were acquired from HeLa cells stably expressing TfnR-EGFP; a three-dimensional image including a metaphase cell was constructed. A still image from Video S1 is shown. (C) Time-lapse analysis of spatiotemporal change in the localization of TfnR-EGFP. HeLa cells expressing TfnR-EGFP were subjected to time-lapse recording using a confocal microscope. Representative frames from Video S2 are shown. (D) Three-dimensional time-lapse analysis of spatiotemporal changes in the localization of TfnR-EGFP. HeLa cells expressing TfnR-EGFP were subjected to time-lapse recording using the A1R-MP confocal microscope. A representative frame from Video $\mathrm{S} 3$ is shown. 

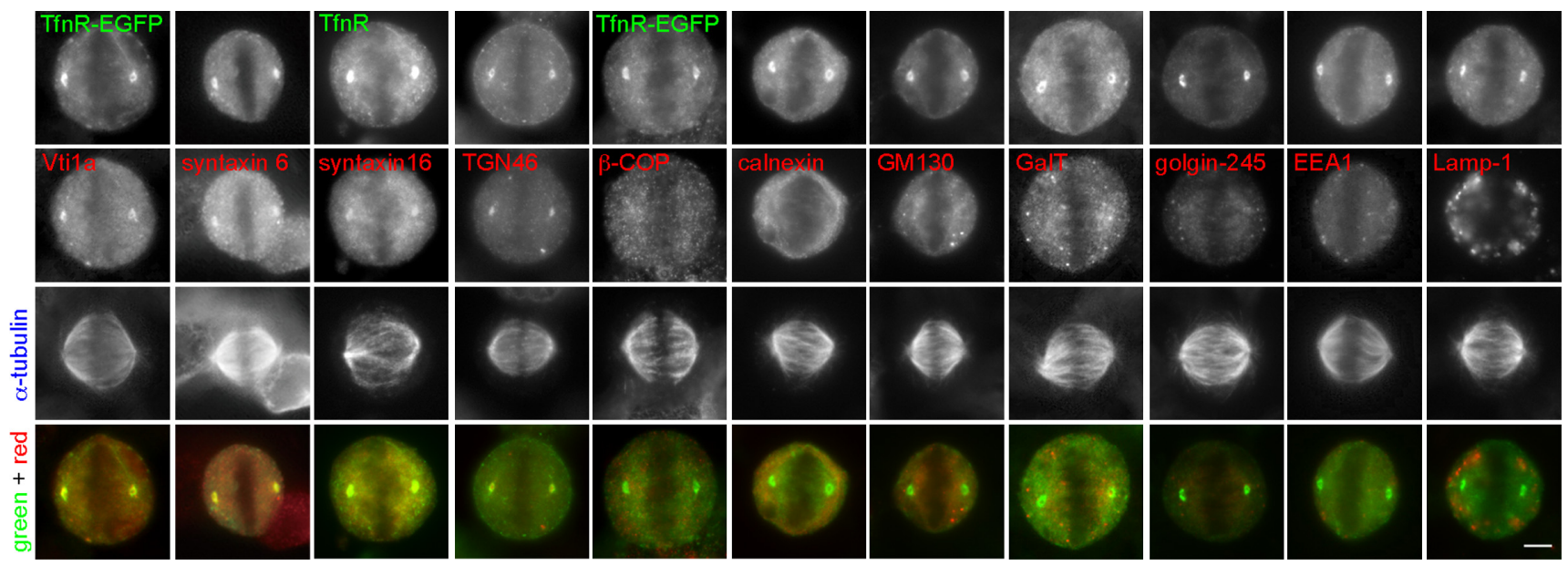

Fig. 2. Distribution of various organellar markers in metaphase cells. HeLa cells were triple-stained for TfnR, $\alpha$-tubulin, and an organellar marker as indicated. HeLa cells expressing TfnR-EGFP were double-stained for $\alpha$-tubulin and an organellar marker indicated. Representative images of metaphase cells are shown. Bar, $5 \mu \mathrm{m}$.

of membrane traffic. AlexaFluor555-Tfn internalized in advance is accessible to the CReSCent structures (Fig. 4A), confirming that the CReSCent represents an endosomal compartment. To examine whether the CReSCent is active in terms of membrane traffic, we performed a FRAP analysis of TfnR-EGFP-expressing cells that had been incubated in the presence of AlexaFluor555-Tfn. As shown in Fig. 4B and Video S4, when the TfnR-EGFP signal on one of the two CReSCent structures in a metaphase cell is photobleached (indicated by an arrow), the TfnR-EGFP signal does not detectably recover, and already internalized AlexaFluor555-Tfn appears to be inaccessible to the CReSCent structure until telophase (circled). Soon after signal recovery, the tight CReSCent structures collapse as TfnREGFP reassembles around the cleavage plane. Furthermore, endocytosed fluorescent $\mathrm{Tfn}$ becomes detectable on the CReSCent structures concurrent with the recovery of the TfnR-EGFP signal (circled). These observations indicate that in early mitotic phases very little, if any, membrane exchange takes place between the CReSCent and other cellular compartments, including the other CReSCent structure on the opposite pole, even though Tfn endocytosis is also reduced in these phases (Schweitzer et al., 2005).

\section{CReSCent consists of two crescent-like clusters of endosomal vesicles}

As shown in Fig. 5A, the CReSCent structures positive for TfnR-EGFP often resemble a ring surrounding each centrosome. These structures are closer to the centrosomes $(\gamma-$ tubulin signals) than NuMA, which is required for focusing the minus ends of spindles (see Fig. 5E). Closer inspection by confocal microscopy and construction of three-dimensional images revealed that the CReSCent is composed of twin crescents around the centrosome (Fig. 5B). To examine the structure at the electron-microscopic level, HeLa cells were allowed to internalize with horseradish peroxidase (HRP)-conjugated Tfn and treated with diaminobenzidine and $\mathrm{H}_{2} \mathrm{O}_{2}$. Tfn-positive vesicles were clustered around the centrosomes as well as scattered throughout the cytoplasm (Fig. 5C). These vesicles were small, with a diameter of approximately $40-60 \mathrm{~nm}$, in good agreement with the diameter of recycling endosomal vesicles determined in a previous study (Sachse et al., 2002). Tfn-positive structures larger than $100 \mathrm{~nm}$ were rarely observed, and adjacent serial sections contained the same vesicle clusters (Fig. 5D), indicating that these vesicles remained clustered without fusing with one another.

\section{Microtubule- and dynein-dependent, centripetal movement of vesicles results in CReSCent formation}

Our immunofluorescence (Fig. 1A) and time-lapse (Fig. 1C and Video S2) analyses suggested that formation of a CReSCent structure initially occurs before separation of duplicated centrosomes. In addition, when TfnR-EGFPexpressing cells were treated with S-trityl-L-cysteine, an inhibitor of Eg5/KIF11, to induce monopolar spindles by inhibiting separation of duplicated centrosomes (Skoufias et al., 2006), TfnR clustering was still evident (Fig. 6A), confirming that formation of bipolar spindles is not a prerequisite for CReSCent formation.

We then set out to investigate what determines positioning of the CReSCent structure around the centrosomes. One possibility is that vesicles accumulate around centrosomes by interacting with either centrosomes per se or pericentrosomal materials. Another possibility is that vesicles accumulate around the centrosome via microtubule-dependent 


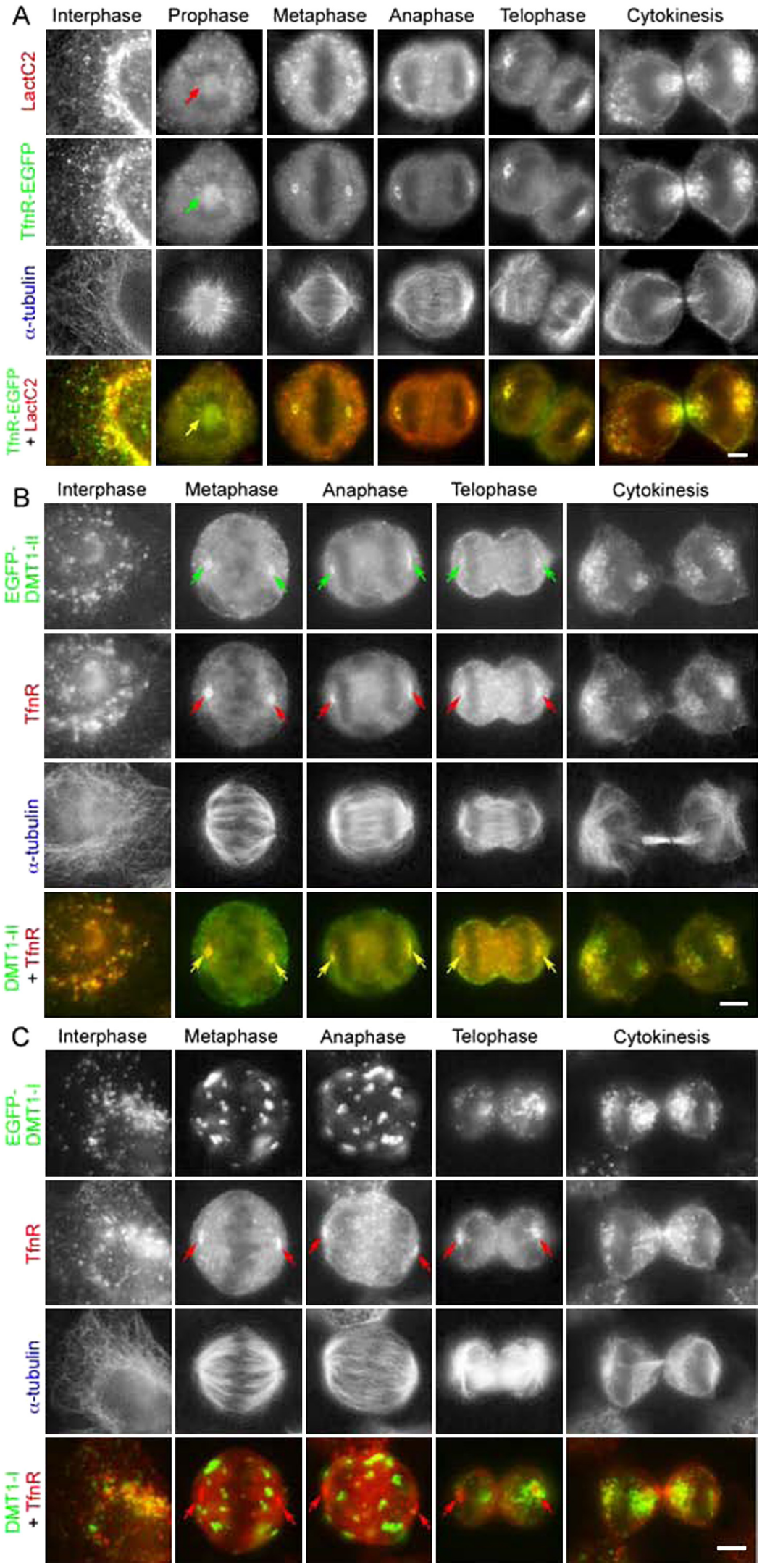

Fig. 3. CReSCent represents an ERC. (A) The CReSCent is a PS-enriched compartment. HeLa cells expressing TfnR-EGFP were fixed, permeabilized, and incubated with recombinant mRFP-LactC2 and anti- $\alpha$-tubulin antibody as described in Materials and Methods. Images were acquired from cells in various mitotic phases and arranged in temporal order. (B, C) Transient localization of DMT1-II, but not DMT1-I, to the CReSCent structures. HeLa cells transfected with an expression vector for EGFP-tagged DMT1-II (B) or DMT1-I (C) were stained for $\mathrm{TfnR}$ and $\alpha$-tubulin. Images were acquired from cells in various cell division phases and arranged in temporal order of the phases. Arrows indicate positions of the CReSCent structures. Bars, $5 \mu \mathrm{m}$. 


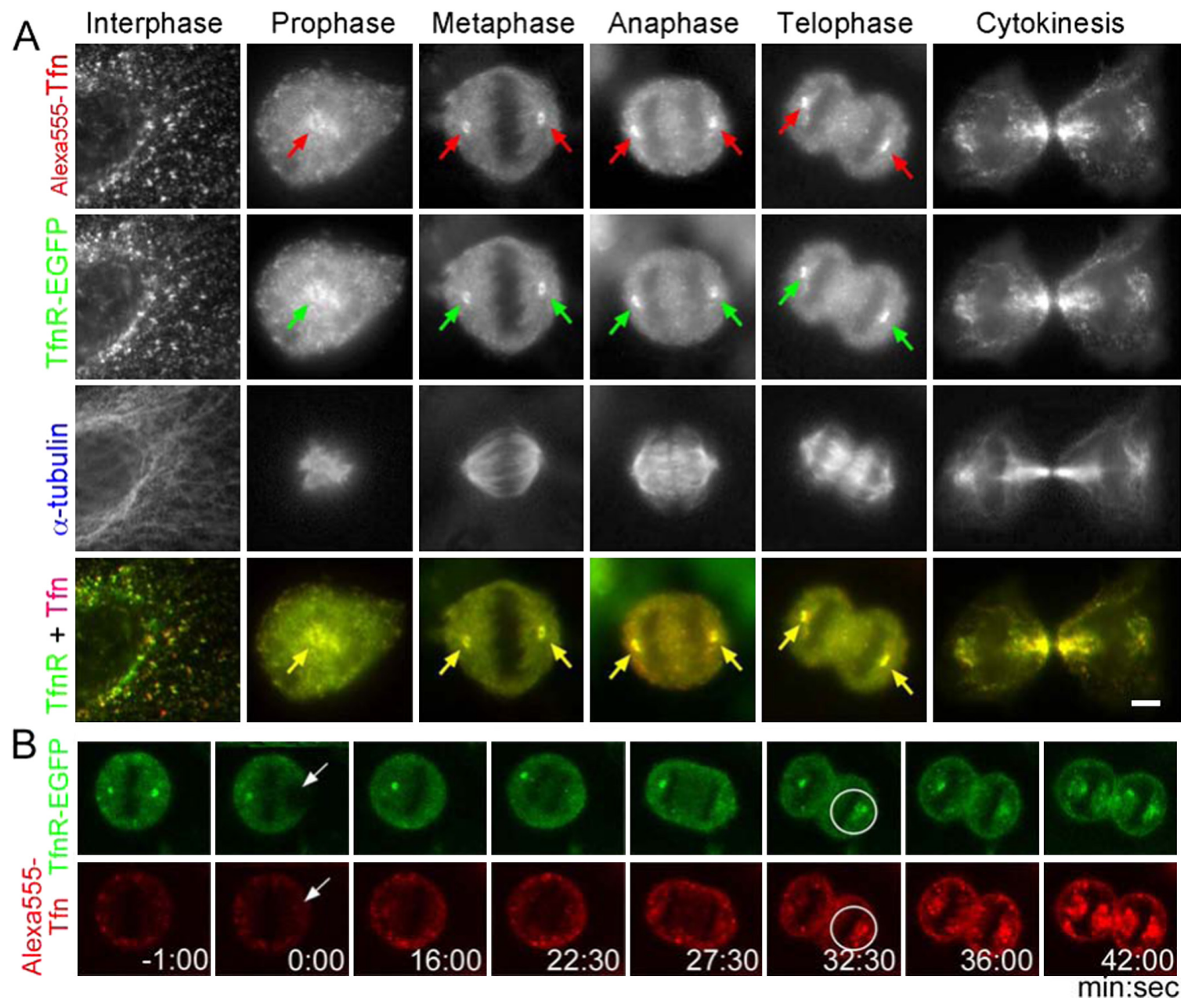

Fig. 4. CReSCent is a recycling compartment that remains inactive until anaphase/telophase. (A) Arrest of Tfn recycling in the CReSCent structures. HeLa cells expressing TfnR-EGFP were subjected to internalization of AlexaFluor555-Tfn and processed for immunofluorescence analysis with anti- $\alpha$ tubulin antibody. Images were acquired from cells in various mitotic phases and arranged in temporal order. Bar, $5 \mu \mathrm{m}$. (B) FRAP analysis of Tfn-EGFP signal around the CReSCent in a cell incubated with AlexaFluor555-Tfn. The signal around one of the two CReSCent structures in a dividing cell was bleached at time $=0$, and fluorescence recovery was followed by time-lapse recording. Representative frames from Video S4 are shown.

movement. To discriminate between these possibilities, we exploited experimental conditions in which varying concentrations of microtubule-depolymerizing drugs, such as nocodazole, arrest mitotic HeLa cells to a similar extent in metaphase, but have differential effects on microtubule organization (Jordan et al., 1992).

When HeLa cells are treated with $0.1 \mu \mathrm{M}$ nocodazole for $16 \mathrm{hr}$, spindles are largely monopolar, and a TfnR-EGFPpositive cluster is formed at the $\gamma$-tubulin-positive center of the spindle assemblies in metaphase-arrested cells (Fig. 6B, left column, and Video S5). After removal of the drug, the single cluster is separated polewards into two CReSCent structures as the cells are released from metaphase arrest (Video S6). When treated with $1.0 \mu \mathrm{M}$ nocodazole, some cells contain several aggregates of short tubulin polymers, and a TfnR-EGFP-positive cluster is formed at the center of each aggregate even though the cluster lacks a $\gamma$-tubulin signal (Fig. 6B, middle column). When cells are treated with $3.3 \mu \mathrm{M}$ or higher concentrations of nocodazole, neither tubulin polymers nor TfnR-EGFP-positive clusters are formed (Fig. 6B, right column, and Video S7). These observations indicate that TfnR-EGFP-positive vesicles accumulate at the minus ends of spindle microtubules, rather than around the centrosome, probably via centripetal movement.

In order to more directly demonstrate the centripetal movement of vesicles, we then performed high-speed and high-resolution analysis using a spinning disc confocal microscope. To this end, we incubated cells with AlexaFluor555Tfn to allow its internalization and followed its movement. As shown in Fig. 6C and Video S8, at the end of G2 phase or in prophase, centripetal movement of Tfn-positive vesicles towards the centrosome position, probably along micro- 

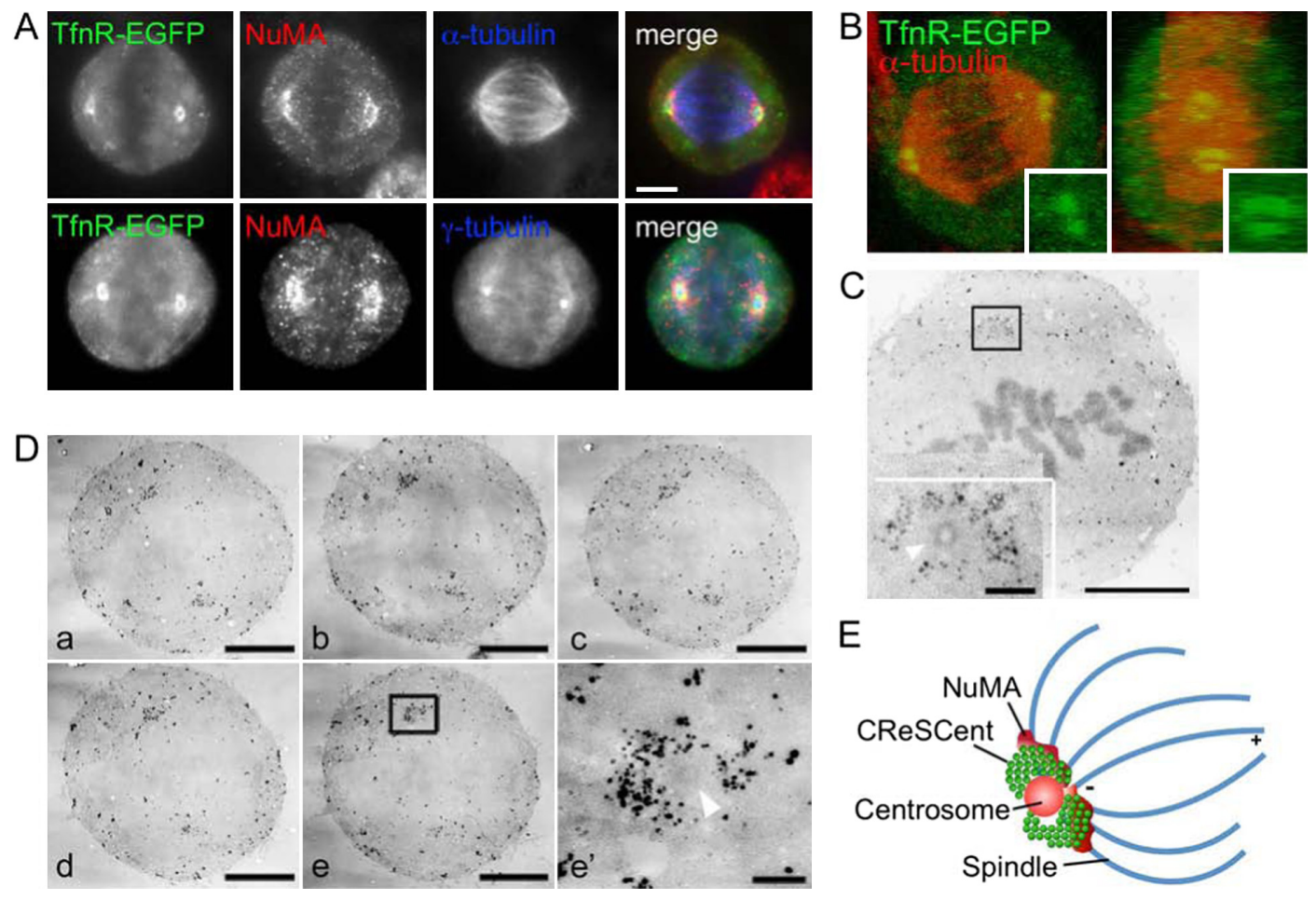

Fig. 5. Confocal and electron microscopic analyses of the CReSCent structure. (A) CReSCent is a ring-like structure around each spindle pole centrosome. HeLa cells expressing TfnR-EGFP were double-stained for NuMA and either $\alpha$-tubulin (upper panels) or $\gamma$-tubulin (lower panels). Representative images of metaphase cells are shown. Bar, $5 \mu \mathrm{m}$. (B) CRESCent are composed of twin crescent-like structures. HeLa cells expressing TfnREGFP were stained for $\alpha$-tubulin and subjected to image acquisition and construction of a three-dimensional image using a PASCAL confocal microscope. (C) Electron micrographs of a HeLa cell, in which HRP-conjugated Tfn was internalized and reacted with diaminobenzidine and $\mathrm{H}_{2} \mathrm{O}_{2}$. Inset shows an enlarged image of the boxed region. White arrowhead indicates a centrosome. Bars, $3 \mu \mathrm{m}$ in the main panel and $0.5 \mu \mathrm{m}$ in the inset. (D) Serial sections of a metaphase cell. Electron micrographs of a cell in which HRP-conjugated Tfn was internalized and reacted with diaminobenzidine and $\mathrm{H}_{2} \mathrm{O}_{2}$. Sections of a representative metaphase cell are shown. Panels a-e are serial sections, and panel e' is an enlarged image of the boxed region in panel e. A white arrowhead indicates a centrosome. Bars, $4 \mu \mathrm{m}$ in a-e; $0.5 \mu \mathrm{m}$ in e'. (E) Schematic representation of relative positioning of centrosome, CReSCent, and spindles.

tubules, is more prevalent than centrifugal movement in the central region of the cell. In contrast, in the peripheral region, the direction of movement is essentially random. Taken together, these data suggest that the CReSCent structure is formed by centripetal/minus end-directed movement along spindle microtubules and subsequent accumulation of endosomal vesicles near centrosomes.

We next examined whether the minus-end directed motor, dynein, is responsible for CReSCent formation. For this purpose, we exploited the fact that overexpression of the dynamitin subunit of the dynactin complex causes disassembly of the complex and results in disruption of dyneindependent maintenance of various compartments in the perinuclear region (Burkhardt et al., 1997; Echeverri et al., 1996). As shown in Fig. 6D and Video S9, cells transfected with an expression vector for FLAG-tagged dynamitin (red arrow) did not form typical CReSCent structures and remained spherical at least $4 \mathrm{hr}$ after the cell had become a spherical shape, while non-transfected cells (blue arrow) completed cytokinesis within $1.5 \mathrm{hr}$ after the cells had become a spherical shape. These observations together indicate that dynein is involved in CReSCent formation.

\section{Discussion}

In this study, we have shown that during mitosis, the ERC exhibits a characteristic spatiotemporal change quite different from that of the Golgi apparatus, particularly in early mitotic phases (Fig. 7). We have also shown that between metaphase and telophase, the ERC forms a tight cluster, which we have designated CReSCent, around each spindle pole centrosome (Fig. 5E and Fig. 7). The CReSCent structure is composed of twin crescent-like clusters of endosomal vesicles (Fig. 5B-E). The CReSCent undergoes minimal membrane exchange with other compartments, including the other CReSCent structure on the opposite pole, until anaphase/telophase, when endocytosed Tfn becomes accessible to it (Fig. 4B and Video S4). During cytokinesis, TfnR, along with endocytosed Tfn, reassembles 

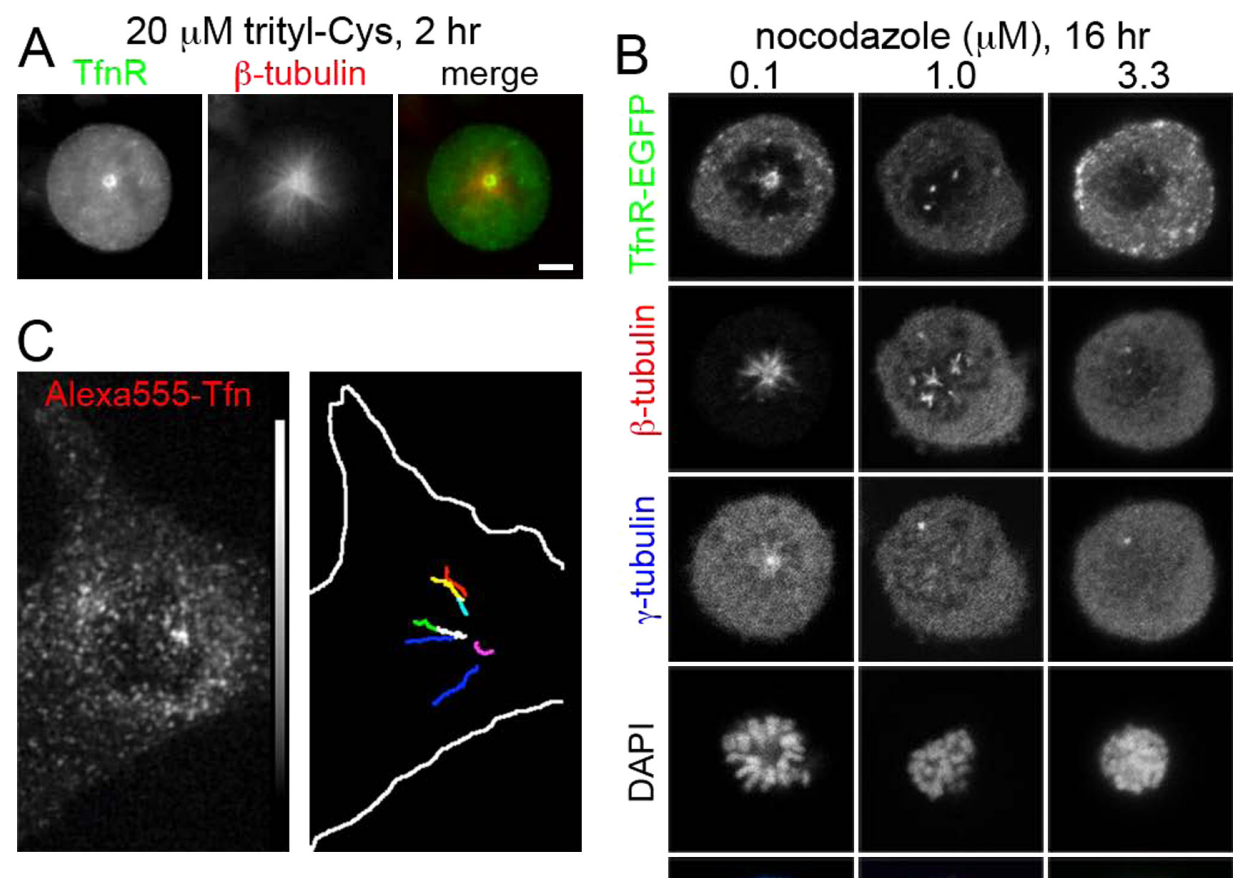

ํํำ
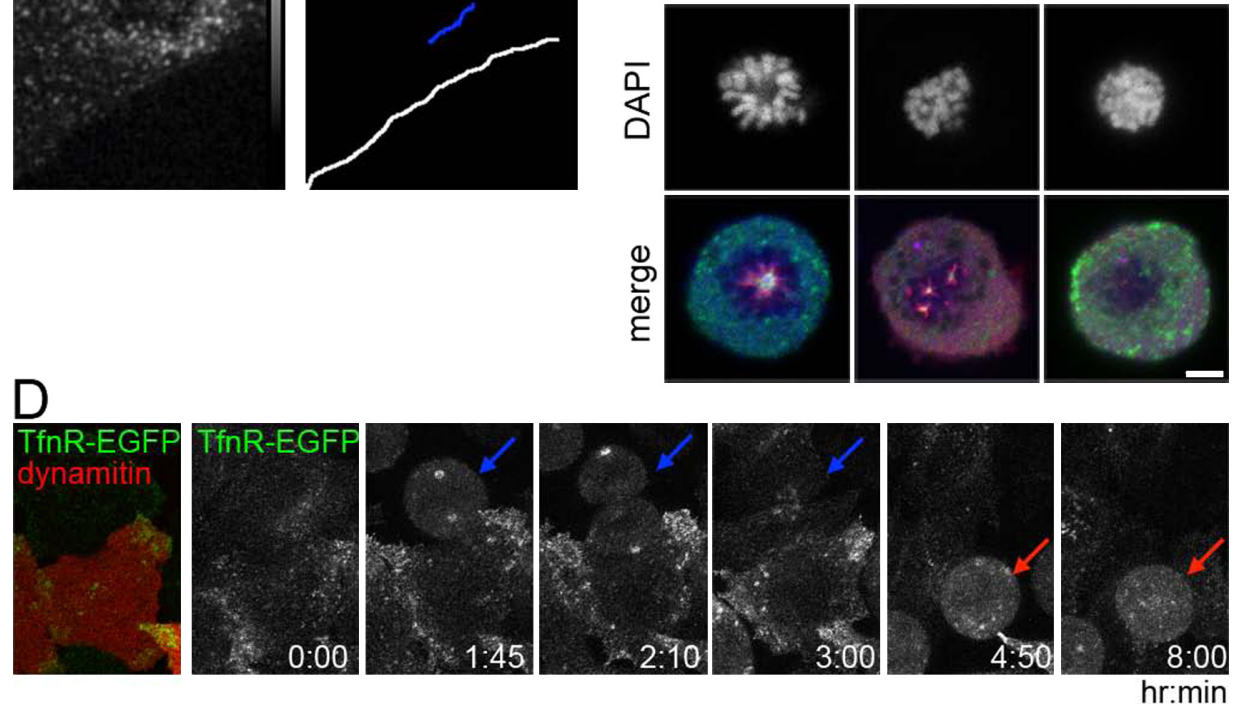

Fig. 6. Microtubule- and dynein-dependent clustering of TfnR. (A) Accumulation of TfnR at the minus ends of monopolar spindles. HeLa cells were treated with $20 \mu \mathrm{M} \mathrm{S}$-trityl-L-cysteine for $2 \mathrm{hr}$ and stained for TfnR and $\beta$-tubulin. (B) Microtubule-dependent formation of CReSCent. HeLa cells expressing TfnR-EGFP were incubated with nocodazole $(0.1,1.0$, or $3.3 \mu \mathrm{M})$ for $16 \mathrm{hr}$, and stained with anti- $\beta$-tubulin, anti- $\gamma$-tubulin and DAPI. (C) Centripetal movement of Tfn-positive vesicles in prophase. HeLa cells incubated with AlexaFluor555-Tfn were observed with a spinning disc microscope. A representative frame from Video S8 is shown (left panel), and movement of representative vesicles in the central region was traced in Video S8 (right panel). Bar, $5 \mu \mathrm{m}$. (D) Overexpression of dynamitin inhibits CReSCent formation and progression of mitosis. HeLa cells stably expressing TfnR-EGFP were transfected with an expression vector for FLAG-tagged dynamitin and an empty mCherry vector to detect transfected cells. The cells were then subjected to time-lapse recording using the A1R-MP confocal microscope. The left panel shows a confocal image before the time-lapse recording, and the next panels show representative frames from Video S9. Red and blue arrows indicate cells with or without transfection of FLAG-dynamitin+mCherry, respectively.

around the furrowing equatorial region (Fig. $4 \mathrm{~A}$ and $4 \mathrm{~B}$, and Video S4), suggesting that delivery of endosomal membranes from the CReSCent to a cellular region where dynamic membrane remodeling occurs may play a critical role in cytokinesis.

Previous work performed more than a quarter century ago suggested that $\mathrm{Tfn}$ recycling is suppressed during mitosis (Sager et al., 1984; Warren et al., 1984). Furthermore, a more recent study showed that suppression of recycling of internalized membranes and proteins during metaphase is well correlated with reduction in the cell surface area and surface expression of membrane proteins (Boucrot and Kirchhausen, 2007). It is therefore likely that the clustered endosomal vesicles in a dividing cell play a fundamental role in restriction of cell surface area by serving as transient membrane reservoirs. Furthermore, the CReSCent structures may play a role in sequestering specific proteins, such as SNAREs (Fig. 2), that are required for membrane deliv- 


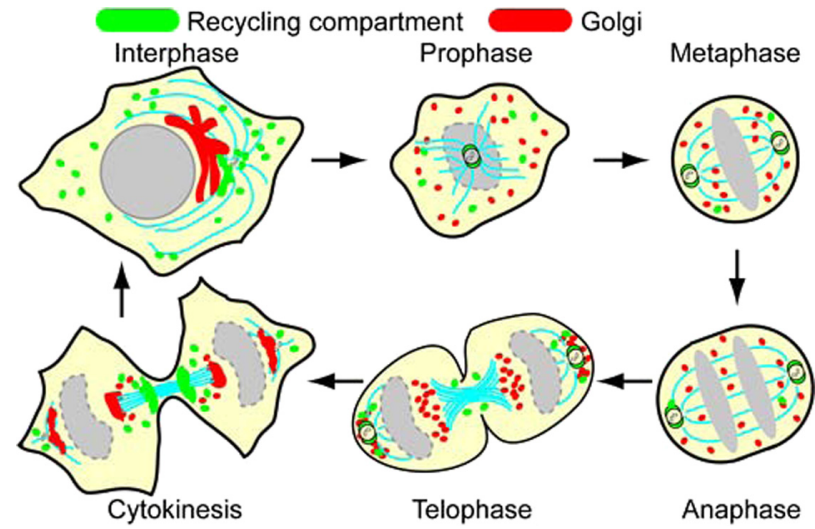

Fig. 7. Schematic representation of spatiotemporal changes in the distribution of the ERC and the Golgi apparatus during mitosis. In interphase HeLa cells, Golgi stacks are localized in the perinuclear region, while the punctate ERC structures are scattered throughout the cytoplasm. In prophase, the Golgi is fragmented or fused with the endoplasmic reticulum, while the ERC starts accumulating around the centrosome. From prometaphase through anaphase, the Golgi remains fragmented, while the ERC clusters around spindle pole centrosomes. In telophase, the Golgi starts forming two assemblies in each dividing part, around spindle pole centrosome and near the central spindle, while the CReSCent structures of the ERC start collapsing. During cytokinesis, the Golgi assemblies are unchanged with respect to the positioning, while the ERC accumulates near the central spindle and within the intercellular bridge. After abscission, two Golgi assemblies are united to form one perinuclear structure, while the ERC is scattered as vesicles throughout the cytoplasm.

ery and/or fusion during late telophase and cytokinesis, when cells undergo dramatic shape changes.

Endosomal vesicles are clustered very transiently at the cell center, within a relatively short period of prophase, and the endosome clustering around the centrated centrosome appears to serve as the cue for subsequent poleward separation of the initial single cluster into two distinct clusters (Fig. 1C and Video S3); this is consistent with a notion that centrosome centration is a fundamental requirement for symmetric division in animal cells (Kimura and Kimura, 2011). Open questions remain regarding how the ERC selectively clusters during mitosis and how membrane traffic is turned on and off at this compartment. In this context, we have shown that, in early mitotic phases, CReSCent is formed in a microtubule- and dynein-dependent manner. Furthermore, we observed, by spinning disc confocal microscopy, bidirectional movement of endosomal vesicles at the end of G2 phase and in prophase, particularly in the peripheral region (Video S8). However, a subpopulation of endosomal vesicles moves in a centripetal manner in the central region (Fig. 6C), although many other vesicles move bidirectionally. In line with our observations, it has recently been reported that a small bias toward centripetal vs. centrifugal movements is sufficient for accumulation of endosomal vesicles around the centrosome (Kimoto et al., 2009).

By contrast, in late mitotic phases, endosomal vesicles positive for $\mathrm{TfnR}$ and endocytosed $\mathrm{Tfn}$ are delivered to the equatorial region (Fig. 4A and 4B, and Video S4), suggesting involvement of plus end-directed kinesin motors. A recent study has provided evidence that switching between kinesin-1 and dynactin-dynein motors controls trafficking of endosomal vesicles in and out of the intercellular bridge and is required for proper cytokinesis (Montagnac et al., 2009). Although bidirectional cargo transport along microtubule tracks is the subject of intense debate and several models have been proposed for regulation of bidirectional organelle motility, plus- and minus-end directed motors on a single cargo work together to switch directions in all the models (Jolly and Gelfand, 2011; Welte, 2010). It is a future issue to be addressed what reciprocally regulates the activities of the plus- and minus-end directed motors during mitosis.

Acknowledgments. We would like to thank Mitsuaki Tabuchi, Miho Ohsugi, and Hiroshi Ohno for critical reading of the manuscript, and Mitsuaki Tabuchi, Tatsuo Suganuma, Minoru Fukuda, Hiroyuki Arai, Roger Tsien, Peter McPherson, Toshiaki Isobe, and Mitsuo Tagaya for kindly providing materials. We also thank Atsuko Yabashi, Katsuyuki Kanno, and Akane Yamada for technical assistance. This work was supported in part by grants from the Ministry of Education, Culture, Sports, Science and Technology of Japan; the Japan Society for Promotion of Science; the Targeted Proteins Research Program; and the Special Coordination Fund for Promoting Science and Technology.

\section{References}

Altan-Bonnet, N., Sougrat, R., and Lippincott-Schwartz, J. 2004. Molecular basis for Golgi maintenance and biogenesis. Curr. Opin. Cell Biol., 16: 364-372.

Boucrot, E. and Kirchhausen, T. 2007. Endosomal recycling controls plasma membrane area during mitosis. Proc. Natl. Acad. Sci. USA, 104: 7939-7944.

Burkhardt, J.K., Echeverri, C.J., Nilsson, T., and Vallee, R.B. 1997. Overexpression of the dynamitin (p50) subunit of the dynactin complex disrupts dynein-dependent maintenance of membrane organelle distribution. J. Cell Biol., 139: 469.

Colanzi, A., Suetterlin, C., and Malhotra, V. 2003. Cell-cycle-specific Golgi fragmentation: how and why? Curr. Opin. Cell Biol., 15: 462467.

Dunster, K., Toh, B.H., and Sentry, J.W. 2002. Early endosomes, late endosomes, and lysosomes display distinct strategies of inheritance with similarities to Golgi-derived membranes. Eur. J. Cell Biol., 81: 117124.

Echeverri, C.J., Paschal, B.M., Vaughan, K.T., and Vallee, R.B. 1996. Molecular characterization of the $50-\mathrm{kD}$ subunit of dynactin reveals function for the complex in chromosome alignment and spindle organization during mitosis. J. Cell Biol., 132: 617-633.

Fürthauer, M. and González-Gaitán, M. 2009. Endocytosis and mitosis: a two-way relationship. Cell Cycle, 8: 3311-3318.

Gaietta, G.M., Giepmans, B.N.G., Deerinck, T.J., Smith, W.B., Ngan, L., Llopis, J., Adams, S.R., Tsien, R.Y., and Ellisman, M.H. 2006. Golgi twins in late mitosis revealed by genetically encoded tags for live cell imaging and correlated electron microscopy. Proc. Natl. Acad. Sci. USA, 103: $17777-17782$.

Ghosh, R.N., Mallet, W.G., Soe, T.T., McGraw, T.E., and Maxfield, F.R. 1998. An endocytosed TGN38 chimeric protein is delivered to the TGN after trafficking through the endocytic recycling compartment in $\mathrm{CHO}$ 
cells. J. Cell Biol., 142: 923-936.

Hirose, H., Arasaki, K., Dohmae, N., Takio, K., Hatsuzawa, K., Nagahama, M., Tani, K., Yamamoto, A., Tohyama, M., and Tagaya, M. 2004. Implication of ZW10 in membrane trafficking between the endoplasmic reticulum and Golgi. EMBO J., 23: 1267-1278.

Ichimura, T., Yamamura, H., Sasamoto, K., Tominaga, Y., Taoka, M., Kakiuchi, K., Shinkawa, T., Takahashi, N., Shimada, S., and Isobe, T. 2005. 14-3-3 proteins modulate the expression of epithelial $\mathrm{Na}^{+}$channels by phosphorylation-dependent interaction with Nedd4-2 ubiquitin ligase. J. Biol. Chem., 280: 13187-13194.

Jolly, A.L. and Gelfand, V.I. 2011. Bidirectional intracelular transport: utility and mechanism. Biochem. Soc. Trans., 39: 1126-1130.

Jordan, M.A., Thrower, D., and Wilson, L. 1992. Effects of vinblastin, podophyllotoxin and nocodazole on mitotic spindles: implications for the role of microtubule dynamics in mitosis. J. Cell Sci., 102: 401-416.

Kain, R., Angata, K., Kerjaschki, D., and Fukuda, M. 1998. Molecular cloning and expression of a novel human trans-Golgi network glycoprotein, TGN51, that contains multiple tyrosine-containing motifs. $J$. Biol. Chem., 273: 981-988.

Kawano, J., Ide, S., Oinuma, T., and Suganuma, T. 1994. A proteinspecific monoclonal antibody to rat liver $\beta 1 \rightarrow 4$ galactosyltransferase and its application to immunohistochemistry. J. Histochem. Cytochem., 42: 363-369.

Kimoto, T., Yamada, M., Ichikawa, T., Honma, D., Cherry, R.J., Morrison, I.E.G., and Kawato, S. 2009. Digital fluorescence analysis of trafficking of single endosomes containing low-density lipoprotein in adrenocortical cells: facilitation of centripetal motion by adrenocorticotropic hormone. Mol. Cell. Endocrinol., 307: 185-195.

Kimura, K. and Kimura A. 2011. A novel mechanism of microtubule length-dependent force to pull centrosomes toward the cell center. BioArchitecture, 1: 74-79.

Kobayashi, T. and Murayama, T. 2009. Cell cycle-dependent microtubulebased dynamic transport of cytoplasmic dynein in mammalian cells. PLoS One, 4: e7827.

Mallard, F., Tang, B.L., Galli, T., Tenza, D., Saint-Pol, A., Yue, X., Antony, C., Hong, W., Goud, B., and Johannes, L. 2002. Early/recycling endosomes-to-TGN transport involves two SNARE complexes and a Rab6 isoform. J. Cell Biol., 156: 653-664.

Maxfield, F.R. and McGraw, T.E. 2004. Endocytic recycling. Nat. Rev Mol. Cell Biol., 5: 121-132.

Montagnac, G., Echard, A., and Chavrier, P. 2008. Endocytic traffic in animal cell cytokinesis. Curr. Opin. Cell Biol., 20: 454-461.

Montagnac, G., Sibarita, J.-B., Loubéry, S., Daviet, L., Romao, M., Raposo, G., and Chavrier, P. 2009. ARF6 interacts with JIP4 to control a motor switch mechanism regulating endosome traffic in cytokinesis. Curr. Biol., 19: 184-195.

Nishimoto-Morita, K., Shin, H.-W., Mitsuhashi, H., Kitamura, M., Zhang, Q., Johannes, L., and Nakayama, K. 2009. Differential effects of depletion of ARL1 and ARFRP1 on membrane trafficking between the transGolgi network and endosomes. J. Biol. Chem., 284: 10583-10592.

Sachse, M., Ramm, G., Strous, G., and Klumperman, J. 2002. Endosomes: multipurpose designs for integrating housekeeping and specialized tasks. Histochem. Cell Biol., 117: 91-104.

Sager, P.R., Brown, P.A., and Berlin, R.D. 1984. Analysis of transferrin recycling in mitotic and interphase HeLa cells by quantitative fluorescence microscopy. Cell, 39: 275-282.
Schweitzer, J.K., Burke, E.E., Goodson, H.V., and D'Souza-Schorey, C. 2005. Endocytosis resumes during late mitosis and is required for cytokinesis. J. Biol. Chem., 280: 41628-41635.

Seemann, J., Pypaert, M., Taguchi, T., Malsam, J., and Warren, G. 2002. Partitioning of the matrix fraction of the Golgi apparatus during mitosis in animal cells. Science, 295: 848-851.

Shin, H.-W., Kobayashi, H., Kitamura, M., Waguri, S., Suganuma, T., Uchiyama, Y., and Nakayama, K. 2005. Roles of ARFRP1 (ADPribosylation factor-related protein 1) in post-Golgi membrane trafficking. J. Cell Sci., 118: 4039-4048.

Shin, H.-W., Morinaga, N., Noda, M., and Nakayama, K. 2004. BIG2, a guanine nucleotide exchange factor for ADP-ribosylation factors: its localization to recycling endosomes and implication in the endosome integrity. Mol. Biol. Cell, 15: 5283-5294.

Shorter, J. and Warren, G. 2002. Golgi architecture and Inheritance. Annu. Rev. Cell Dev. Biol., 18: 379-420.

Simon, G.C., Schonteich, E., Wu, C.C., Piekny, A., Ekiert, D., Yu, X., Gould, G.W., Glotzer, M., and Prekeris, R. 2008. Sequential Cyk-4 binding to ECT2 and FIP3 regulates cleavage furrow ingression and abscission during cytokinesis. EMBO J., 27: 1791-1803.

Skoufias, D.A., DeBonis, S., Saoudi, Y., Lebeau, L., Crevel, I., Cross, R., Wade, R.H., Hackney, D., and Kozielski, F. 2006. S-Trityl-L-cysteine is a reversible, tight binding inhibitor of the human kinesin Eg5 that specifically blocks mitotic progression. J. Biol. Chem., 281: 17559-17569.

Tabuchi, M., Tanaka, N., Nishida-Kitayama, J., Ohno, H., and Kishi, F. 2002. Alternative splicing regulates the subcellular localization of divalent metal transporter 1 isoforms. Mol. Biol. Cell, 13: 4371-4387.

Takahashi, S., Kubo, K., Waguri, S., Yabashi, A., Shin, H.-W., Katoh, Y., and Nakayama, K. 2012. Rab11 regulates exocytosis of recycling vesicles at the plasma membrane. J. Cell Sci., 125: 4049-4057.

Thomas, S., Ritter, B., Verbich, D., Sanson, C., Bourbonnière, L., McKinney, R.A., and McPherson, P.S. 2009. Intersectin regulates dendritic spine development and somatodendritic endocytosis but not synaptic vesicle recycling in hippocampal neurons J. Biol. Chem., 284: 12410-12419.

Uchida, Y., Hasegawa, J., Chinnapen, D., Inoue, T., Okazaki, S., Kato, R., Wakatsuki, S., Misaki, R., Koike, M., Uchiyama, Y., Iemura, S.-I., Natsume, T., Kuwahara, R., Nakagawa, T., Nishikawa, K., Mukai, K., Miyoshi, E., Taniguchi, N., Sheff, D., Lencer, W.I., Taguchi, T., and Arai, H. 2011. Intracellular phosphatidylserine is essential for retrograde membrane traffic through endosomes. Proc. Natl. Acad. Sci. USA, 108: 15846-15851

Waguri, S., Kohmura, M., Gotow, T., Watanabe, T., Ohsawa, Y., Kominami, E., and Uchiyama, Y. 1999. The induction of autophagic vacuoles and the unique endocytic compartments, C-shaped multivesicular bodies, in $\mathrm{GH}_{4} \mathrm{C}_{1}$ cells after treatment with $17 \beta$-estradiol, insulin and EGF. Arch. Histol. Cytol., 62: 423-434.

Warren, G., Davoust, J., and Cockcroft, A. 1984. Recycling of transferrin receptors in A431 cells is inhibited during mitosis. EMBO J., 3: 22172225 .

Wei, J.-H. and Seemann, J. 2009. Mitotic division of the mammalian Golgi apparatus. Sem. Cell Dev. Biol., 20: 810-816.

Welte, M.A. 2010. Bidirectional transport: matchmaking for motors. Curr. Biol., 20: R410-R413.

(Received for publication, December 3, 2012, accepted, January 10, 2013 and published online, January 16, 2013) 